\title{
Searching for Gravitational Waves from Cosmological Phase Transitions with the NANOGrav 12.5-Year Dataset
}

Zaven Arzoumanian, ${ }^{1}$ Paul T. Baker, ${ }^{2}$ Harsha Blumer, ${ }^{3,4}$ Bence Bécsy, ${ }^{5}$ Adam Brazier, ${ }^{6,7}$ Paul R. Brook, ${ }^{3,4}$ Sarah Burke-Spolaor, ${ }^{3,4,8}$ Maria Charisi, ${ }^{9}$ Shami Chatterjee, ${ }^{6}$ Siyuan Chen, ${ }^{10,11,12}$ James M. Cordes, ${ }^{6}$ Neil J. Cornish, ${ }^{5}$ Fronefield Crawford, ${ }^{13}$ H. Thankful Cromartie, ${ }^{6}$ Megan E. DeCesar, ${ }^{14,15^{*}}$ Paul B. Demorest, ${ }^{16}$ Timothy Dolch, ${ }^{17,18}$ Justin A. Ellis, ${ }^{19}$ Elizabeth C. Ferrara, ${ }^{20,21,22}$ William Fiore, ${ }^{3,4}$ Emmanuel Fonseca, ${ }^{23}$ Nathan Garver-Daniels, ${ }^{3,4}$ Peter A. Gentile, ${ }^{3,4}$ Deborah C. Good, ${ }^{24}$ Jeffrey S. Hazboun, ${ }^{25}$ A. Miguel Holgado, ${ }^{26,27}$ Kristina Islo, ${ }^{28}$ Ross J. Jennings, ${ }^{6}$ Megan L. Jones, ${ }^{28}$ Andrew R. Kaiser, ${ }^{3,4}$ David L. Kaplan, ${ }^{28}$ Luke Zoltan Kelley, ${ }^{29}$ Joey Shapiro Key, ${ }^{25}$ Nima Laal, ${ }^{30}$ Michael T. Lam, ${ }^{31,32}$ T. Joseph W. Lazio, ${ }^{33}$ Vincent S. H. Lee, ${ }^{34}$ Duncan R. Lorimer, ${ }^{3,4}$ Jing Luo, ${ }^{35}$ Ryan S. Lynch, ${ }^{36}$

Dustin R. Madison, ${ }^{3,4}$ Maura A. McLaughlin, ${ }^{3,4}$ Chiara M. F. Mingarelli, ${ }^{37,38}$ Andrea Mitridate $\odot,{ }^{34} \mathrm{Cherry} \mathrm{Ng},{ }^{39}$ David J. Nice, ${ }^{14}$ Timothy T. Pennucci, ${ }^{40,41}$ Nihan S. Pol, ${ }^{3,4,9}$ Scott M. Ransom, ${ }^{40}$ Paul S. Ray, ${ }^{42}$ Brent J. Shapiro-Albert, ${ }^{3,4}$ Xavier Siemens, ${ }^{30,28}$ Joseph Simon, ${ }^{33,43}$ Renée Spiewak, ${ }^{44}$ Ingrid H. Stairs,${ }^{24}$ Daniel R. Stinebring, ${ }^{45}$ Kevin Stovall, ${ }^{16}$ Jerry P. Sun, ${ }^{30}$ Joseph K. Swiggum, ${ }^{14}$ Stephen R. Taylor, ${ }^{9}$ Jacob E. Turner, ${ }^{3,4}$ Michele Vallisneri, ${ }^{33}$ Sarah J. Vigeland, ${ }^{28}$ Caitlin A. Witt, ${ }^{3,4}$ and Kathryn M. Zurek ${ }^{34}$

(NANOGrav Collaboration)

\footnotetext{
${ }^{1}$ X-Ray Astrophysics Laboratory, NASA Goddard Space Flight Center, Code 662, Greenbelt, Maryland 20771, USA

${ }^{2}$ Department of Physics and Astronomy, Widener University, One University Place, Chester, Pennsylvania 19013, USA

${ }^{3}$ Department of Physics and Astronomy, West Virginia University, P.O. Box 6315, Morgantown, West Virginia 26506, USA

${ }^{4}$ Center for Gravitational Waves and Cosmology, West Virginia University, Chestnut Ridge Research Building, Morgantown, West Virginia 26505, USA

${ }^{5}$ Department of Physics, Montana State University, Bozeman, Montana 59717, USA

${ }^{6}$ Cornell Center for Astrophysics and Planetary Science and Department of Astronomy, Cornell University, Ithaca, New York 14853, USA

${ }^{7}$ Cornell Center for Advanced Computing, Cornell University, Ithaca, New York 14853, USA

${ }^{8}$ CIFAR Azrieli Global Scholars program, CIFAR, Toronto, Canada

${ }^{9}$ Department of Physics and Astronomy, Vanderbilt University, 2301 Vanderbilt Place, Nashville, Tennessee 37235, USA

${ }^{10}$ Station de Radioastronomie de Nancay, Observatoire de Paris, Universite PSL, CNRS, Universite d'Orleans, 18330 Nancay, France

${ }^{11}$ FEMTO-ST Institut de recherche, Department of Time and Frequency, UBFC and CNRS, ENSMM, 25030 Besancon, France

${ }^{12}$ Laboratoire de Physique et Chimie de l'Environment et de l'Espace, LPC2E UMR7328, Universite d'Orleans, CNRS, 45071 Orleans, France

${ }^{13}$ Department of Physics and Astronomy, Franklin \& Marshall College, P.O. Box 3003, Lancaster, Pennsylvania 17604, USA

${ }^{14}$ Department of Physics, Lafayette College, Easton, Pennsylvania 18042, USA

${ }^{15}$ George Mason University, Fairfax, Virginia 22030, USA

${ }^{16}$ National Radio Astronomy Observatory, 1003 Lopezville Road, Socorro, New Mexico 87801, USA

${ }^{17}$ Department of Physics, Hillsdale College, 33 E. College Street, Hillsdale, Michigan 49242, USA

${ }^{18}$ Eureka Scientific, Inc. 2452 Delmer Street, Suite 100, Oakland, California 94602-3017

${ }^{19}$ Infinia ML, 202 Rigsbee Avenue, Durham, North Carolina 27701, USA

${ }^{20}$ Department of Astronomy, University of Maryland, College Park, Maryland 20742, USA

${ }^{21}$ Center for Research and Exploration in Space Science and Technology, NASA/GSFC, Greenbelt, Maryland 20771, USA

${ }^{22}$ NASA Goddard Space Flight Center, Greenbelt, Maryland 20771, USA

${ }^{23}$ Department of Physics, McGill University, 3600 University Street, Montreal, Quebec H3A 2T8, Canada

${ }^{24}$ Department of Physics and Astronomy, University of British Columbia,

6224 Agricultural Road, Vancouver, British Columbia V6T 1Z1, Canada

${ }^{25}$ University of Washington Bothell, 18115 Campus Way NE, Bothell, Washington 98011, USA

${ }^{26}$ Department of Astronomy and National Center for Supercomputing Applications, University of Illinois at Urbana-Champaign, Urbana, Illinois 61801, USA

${ }^{27}$ McWilliams Center for Cosmology and Department of Physics, Carnegie Mellon University, Pittsburgh, Pennsylvania 15213, USA

${ }^{28}$ Center for Gravitation, Cosmology and Astrophysics, Department of Physics, University of Wisconsin-Milwaukee, P.O. Box 413, Milwaukee, Wisconsin 53201, USA

${ }^{29}$ Center for Interdisciplinary Exploration and Research in Astrophysics (CIERA), Northwestern University, Evanston, Illinois 60208, USA

${ }^{30}$ Department of Physics, Oregon State University, Corvallis, Oregon 97331, USA
} 


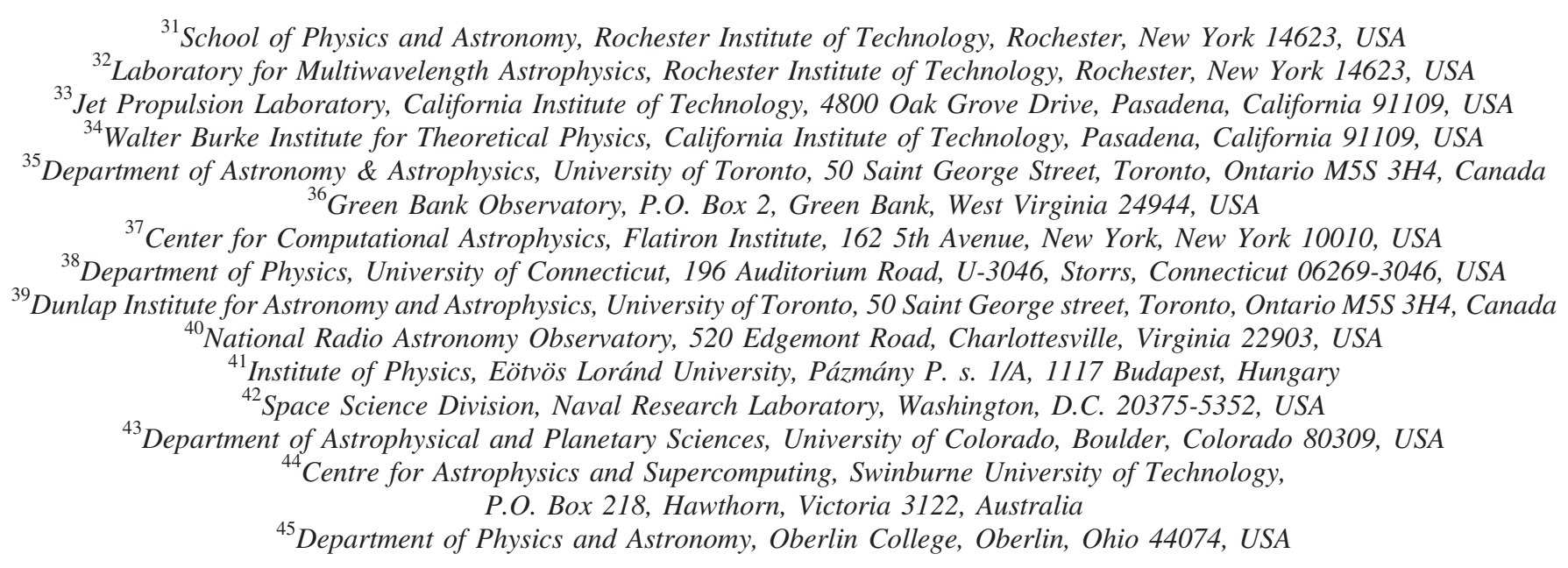

(Received 28 April 2021; revised 17 September 2021; accepted 11 November 2021; published 15 December 2021)

\begin{abstract}
We search for a first-order phase transition gravitational wave signal in 45 pulsars from the NANOGrav 12.5-year dataset. We find that the data can be modeled in terms of a strong first order phase transition taking place at temperatures below the electroweak scale. However, we do not observe any strong preference for a phase-transition interpretation of the signal over the standard astrophysical interpretation in terms of supermassive black hole mergers; but we expect to gain additional discriminating power with future datasets, improving the signal to noise ratio and extending the sensitivity window to lower frequencies. An interesting open question is how well gravitational wave observatories could separate such signals.
\end{abstract}

DOI: 10.1103/PhysRevLett.127.251302

Introduction.-The search for gravitational waves (GWs) spans many orders of magnitude and encapsulates a plethora of source phenomena. At very low frequencies $(\sim 1-100 \mathrm{nHz})$, pulsar-timing arrays (PTAs; [1-3]) aim to detect GWs through the presence of correlated deviations to radiopulse arrival times across an ensemble of precisely timed Milky Way millisecond pulsars. There are three PTA collaborations that currently have decadal-length timing data from an ensemble of pulsars: The North American Nanohertz Observatory for Gravitational Waves (NANOGrav; [4]), the European Pulsar Timing Array (EPTA; [5]), and the Parkes Pulsar Timing Array (PPTA; [6]). These three, in addition to the Indian PTA (InPTA; [7]), are synthesized into the International Pulsar Timing Array (IPTA; Perera et al. [8]). There are also emerging efforts in China (CPTA; [9]), as well as some telescope-centered timing programs (MeerKAT; [10]; CHIME; [11]).

The dominant $\mathrm{GW}$ signals at such low frequencies are expected to be from a cosmic population of tightly bound inspiraling supermassive binary black holes (SMBHBs; $[12,13])$, producing an aggregate incoherent signal that we search for as a stochastic GW background (GWB), and also individual binary signals that we attempt to resolve out of this stochastic confusion background. However, other more speculative GW sources in the PTA frequency range include cosmic strings [14,15], a primordial GWB produced by quantum fluctuations of the gravitational field in the early universe, amplified by inflation [16-18], and cosmological phase transitions [19-23], the latter of which is the subject of this study.

The most recent PTA results are from NANOGrav's analysis of 12.5 years of precision timing data from 47 pulsars [ [24], hereafter NG12], of which 45 exceeded a timing baseline of 3 years and were analyzed in a search for a stochastic GWB [ [25], hereafter NG12gwb]. NANOGrav reported strong evidence for a common-spectrum lowfrequency stochastic process in its array of 45 analyzed pulsars, where $\sim 10$ of those pulsars are strongly supportive, most are ambivalent, and a few seem to disfavor the process (although not significantly). No evidence for the characteristic interpulsar correlation signature imparted by GWs was found. At low frequencies the shape of the characteristic strain spectrum was well matched to a power law, with an amplitude and slope consistent with theoretical models of SMBHB populations. Under a model that assumes the origin of the GWB is a population of SMBHBs, the median characteristic strain amplitude at a frequency of $1 /$ year is $1.92 \times 10^{-15}$. Interpretations of this common-spectrum process as a GWB from SMBHBs have since appeared in the literature, showing that, if it is indeed so, robust evidence of the distinctive interpulsar correlations should accrue within the next several years, followed by characterization of the strain spectrum and astrophysical probes of the underlying population [26,27]. However, the Bayesian 
posterior probability distributions of the strain-spectrum amplitude and slope are broad enough to entertain a variety of different source interpretations, many of which have since appeared in the literature [e.g., [28-31] ].

In this Letter we consider gravitational waves produced by first-order cosmological phase transitions, both as an alternative origin of the common process measured in the NANOGrav 12.5-year dataset [32-39], and as a subdominant signal to that produced by SMBHBs. The frequency range to which NANOGrav is sensitive corresponds to phase transitions at temperatures below the electroweak phase transitions of the standard model (i.e., $T \lesssim$ $100 \mathrm{GeV})$. This has led many to consider higher frequency GW observatories, such as LISA and LIGO, as the dominant instruments to search for phase transitions. However, phase transitions may occur at much lower temperatures in particular in hidden sectors [40-42]. Hidden sectors feature rich dynamics, with multiple matter fields and forces, independent of the dynamics of the standard model. They appear generically in top-down constructions like string theory, and in some solutions to the so-called hierarchy problem. In many cases, they may be difficult to detect via their particle interactions with the standard model, but gravity is an irreducible messenger. In this regard, PTAs provide a powerful complementary probe to the dynamics of hidden sectors already being explored through many terrestrial, astrophysical, and cosmological probes (see Ref. [43] for a recent summary).

Previous studies on cosmological first order phase transition in the context of the NANOGrav results were carried out in $[33,37,44,45]$. Our analysis presents two main novelties compared to these works: first, we properly include the relevant noise sources and discuss the impact of backgrounds (like the one generated by SMBHBs); second, we discuss how the results are affected by the theoretical uncertainties on the GW spectrum produced by first order phase transitions.

The outline of this Letter is as follows. In the next section we briefly summarize the signature of GWs from the dominant background of SMBH mergers. We then dive into the main subject of this Letter, GWs from a first-order phase transition, where we discuss the relevant parameters characterizing the signal. We then carry out an analysis with the NANOGrav 12.5-year dataset, finding that the data can be modeled in terms of a strong phase transition with a transition temperature around $10 \mathrm{MeV}$. The dataset and data model for these analyses are exactly as described in NG12 and NG12gwb, respectively. All common processes (whether interpreted as being of SMBHB or phase-transition origin) are modeled within the five lowest sampling frequencies of the array time series, corresponding to $\sim 2.5-12 \mathrm{nHz}$. Finally, we discuss theoretical uncertainties, and compare the PT interpretation of the data to the standard interpretation in terms of SMBHBs finding no strong preference for one over the other.
GWs from SMBHB mergers.-Regardless of origin, the energy density of GWs as a fraction of closure density is related to the $\mathrm{GW}$ characteristic strain spectrum by [46]

$$
\Omega_{\mathrm{GW}}(f)=\frac{2 \pi^{2}}{3 H_{0}^{2}} f^{2} h_{c}^{2}(f)
$$

where $H_{0}$ is the Hubble constant (set here to be $67 \mathrm{~km} / \mathrm{s} / \mathrm{Mpc}$ [47]), and the GWB characteristic strain spectrum $h_{c}(f)$ is often described by a power-law function for astrophysical and cosmological sources:

$$
h_{c}(f)=A_{\mathrm{GWB}}\left(\frac{f}{\mathrm{yr}^{-1}}\right)^{\alpha},
$$

where $A_{\mathrm{GWB}}$ is the amplitude at a reference frequency of $1 /$ year, and $\alpha$ is an exponent that depends on the origin of the GWB. For a population of inspiraling SMBHBs, this is $\alpha=-2 / 3$ [48]. The cross-power spectral density of GWinduced timing deviations between two pulsars $a$ and $b$ can be written as

$$
S_{a b}(f)=\Gamma_{a b} \frac{A_{\mathrm{GWB}}^{2}}{12 \pi^{2}}\left(\frac{f}{\mathrm{yr}^{-1}}\right)^{-\gamma} \mathrm{yr}^{3},
$$

where $\gamma \equiv 3-2 \alpha=13 / 3$ for SMBHBs, and $\Gamma_{a b}$ is the Hellings-Downs [49] correlation coefficient between pulsar $a$ and pulsar $b$.

GWs from first-order phase transition.-A first-order phase transition (PT) occurs when the true minimum of a potential is separated from a false minimum by a barrier through which a field must locally tunnel. This can occur in either weakly coupled (where a scalar field tunnels) or strongly coupled (where a vacuum condensate corresponds to the scalar field) theory. Such transitions are known to proceed through nucleation of bubbles of true vacuum which, if sufficiently large, expand in the background plasma (still in the false vacuum). Collisions of these bubbles, as well as interactions between the expanding bubble walls and the surrounding plasma, can be efficient sources of GWs.

We characterize the phase transition in terms of four parameters: (1) $T_{*}$ - The Universe temperature at which the phase transition takes place. (2) $\alpha_{*}$-The strength of the phase transition, defined as the ratio of the vacuum and relativistic energy density at the time of the phase transition. (3) $\beta / H_{*}$-The bubble nucleation rate in units of the Hubble rate at the time of the phase transition, $H_{*}$. (4) $v_{w}$ The velocity of the bubble walls.

The three main sources of GWs associated with a firstorder phase transition are (i) collisions of bubble walls, (ii) collisions of the sound waves generated in the background plasma by the bubbles expansion, and (iii) turbulence in the plasma generated by expansion and collisions of the sound wave. However, in this analysis we will not 
TABLE I. Parameters for the gravitational wave spectrum of Eq. (4). The values of the parameters $(a, b, c)$ in the spectral shape of the bubble contribution are reported in Table II.

\begin{tabular}{lcc}
\hline \hline & Bubbles [58] & Sound waves [59] \\
\hline$\Delta\left(v_{w}\right)$ & {$\left[0.48 v_{w}^{3} /\left(1+5.3 v_{w}^{2}+5 v_{w}^{4}\right)\right]$} & $0.513 v_{w}$ \\
$\kappa$ & $\kappa_{\phi}$ & $\kappa_{\mathrm{SW}}$ \\
$p$ & 2 & 2 \\
$q$ & 2 & 1 \\
$\mathcal{S}(x)$ & $\left\{(a+b)^{c} /\left[b x^{-a / c}+a x^{b / c}\right]^{c}\right\}$ & $x^{3}\left[7 /\left(4+3 x^{2}\right)\right]^{7 / 2}$ \\
$f_{*} / \beta$ & {$\left[0.35 /\left(1+0.07 v_{w}+0.69 v_{w}^{4}\right)\right]$} & $\left(0.536 / v_{w}\right)$ \\
\hline \hline
\end{tabular}

include the turbulence contribution as it usually is subleading compared to the sound-wave one, and also affected by the largest theory uncertainties (see for example [50-52] for recent developments).

The contribution to the total GW spectrum from bubbles and sound waves collisions can be parametrized as [50,53]

$$
h^{2} \Omega(f)=\mathcal{R} \Delta\left(v_{w}\right)\left(\frac{\kappa \alpha_{*}}{1+\alpha_{*}}\right)^{p}\left(\frac{H_{*}}{\beta}\right)^{q} \mathcal{S}\left(f / f_{*}^{0}\right),
$$

where the prefactor $\mathcal{R} \simeq 7.69 \times 10^{-5} g_{*}^{-1 / 3}$ accounts for the redshift of the GW energy density, $\mathcal{S}(\cdots)$ parametrizes the spectral shape, and $\Delta\left(v_{w}\right)$ is a normalization factor which depends on the bubble wall velocity, $v_{w}$. The value of the peak frequency today, $f_{*}^{0}$, is related to the value of the peak frequency at emission, $f_{*}$, by

$f_{*}^{0} \simeq 1.13 \times 10^{-10} \mathrm{~Hz}\left(\frac{f_{*}}{\beta}\right)\left(\frac{\beta}{H_{*}}\right)\left(\frac{T_{*}}{\mathrm{MeV}}\right)\left(\frac{g_{*}}{10}\right)^{1 / 6}$,

where $g_{*}$ denotes the number of relativistic degrees of freedom at the time of the phase transition. The values of the peak frequency at emission, the spectral shape, the normalization factor, and the exponents $p$ and $q$ are reported in Table I for all the production mechanisms considered in this work. Because of the finite lifetime $[54,55]$ of the sound waves, to derive $\Omega_{\mathrm{SW}} \mathrm{Eq}$. (4) needs to be multiplied by a suppression factor $\Upsilon\left(\tau_{\mathrm{SW}}\right)$ given by [54]

$$
\Upsilon\left(\tau_{\mathrm{SW}}\right)=1-\left(1+2 \tau_{\mathrm{SW}} H_{*}\right)^{-1 / 2}
$$

where the sound-wave lifetime is usually taken to be the timescale for the onset of turbulent behaviors in the plasma [56]: $\tau_{\mathrm{SW}} \approx R_{*} / \bar{U}_{f}$, where the average bubble separation is given by $R_{*}=(8 \pi)^{1 / 3} \beta^{-1} \max \left(v_{w}, c_{s}\right)$ [57], and $\bar{U}_{f}^{2} \approx$ $3 \kappa_{\mathrm{SW}} \alpha /\left[4\left(1+\alpha_{*}\right)\right][56]$.

Generally both production mechanisms contribute to the GW spectrum. However, if the bubble walls interact with the surrounding plasma most of the energy released in the PT is expected to be transferred to the plasma so that the sound waves' (and possibly the turbulence) contribution dominates the GW spectrum. An exception to this scenario is provided by models in which the bubble walls do not interact with the plasma, or by models where the energy released in the PT is large enough that the friction exerted by the plasma is not enough to stop the walls from keep accelerating (runaway scenario). However, determining wether or not the runaway regime is realized is either model dependent or affected by large theoretical uncertainties. Therefore, we perform two separate analyses. (1) A sound-wave-only (SWO) analysis, where we assume that the runaway regime is not reached and that the sound wave and turbulence contributions dominate the GW spectrum; therefore we set $\kappa_{\phi}=0$, and use the results of reference [60] to derive $\kappa_{\mathrm{SW}}$ as a function of $v_{w}$ and $\alpha_{*}$. (2) A bubble-only (BO) analysis, where we assume that the runaway regime is reached and that bubble collisions dominate the $\mathrm{GW}$ spectrum; we then set $v_{w}=1, \kappa_{\mathrm{SW}}=0$ and $\kappa_{\phi}=1$.

We conclude this section emphasizing that, despite recent progress, large theoretical uncertainties still affect the prediction of the GW signal produced in cosmological phase transitions. To get an idea of the impact that these uncertainties have on our results we will study how the BO analysis is impacted by them. Similar, if not larger, uncertainties affect the sound wave contribution and would impact the results of the SWO analysis.

Assuming that the stress energy density of the expanding bubbles is localized in an infinitesimally thin shell near the bubble wall (thin shell approximation), and that it instantaneously decays to zero after two bubbles collide (envelope approximation), the bubble spectral shape can be derived analytically $[58,61]$. The spectral shape parameters obtained in this way are reported in the left column of Table II. To go beyond these approximations, 3D lattice simulations are needed. These simulations are extremely expensive given the hierarchy between the large simulation volume needed to include multiple bubbles, and the small lattice spacing needed to resolve the thin walls. Because of the relativistic contraction of the wall width, this separation of scales becomes increasingly large for increasing wall velocities, making it impossible to simulate ultrarelativistic walls. However, the GW spectrum can be simulated at

TABLE II. Comparison of the bubble spectral shape parameters derived using the envelope and thin wall approximation [58] (left column), the semianalytic approach of reference [64] (middle column), and lattice simulations [62] (right column). For numerical and semianalytic results the values of the parameters depend on the choice of the scalar field potential; we report the range of values obtained for the different scalar field potentials considered in the above mentioned works.

\begin{tabular}{lccc}
\hline \hline & Envelope & Semianalytic & Numerical \\
\hline$a$ & 3 & $1-2.2$ & $1.6-0.7$ \\
$b$ & 1 & $2.6-2.9$ & $1.4-2.3$ \\
$c$ & 1.5 & $1.5-3.5$ & 1 \\
$f_{*} / \beta$ & {$\left[0.35 /\left(1+0.07 v_{w}+0.69 v_{w}^{2}\right)\right]$} & 0.1 & 0.2 \\
\hline \hline
\end{tabular}


TABLE III. Prior distributions for the parameters used in all the analyses in this work. The prior for the bubble wall velocity reported in this table is the one used for the SWO analysis, for the BO analyses we use $v_{w}=1$ as explained in the text.

\begin{tabular}{|c|c|c|c|}
\hline Parameter & Description & Prior & Comments \\
\hline \multicolumn{4}{|c|}{ White noise } \\
\hline$E_{k}$ & EFAC per backend-receiver system & Uniform $[0,10]$ & Single-pulsar analysis only \\
\hline$Q_{k}^{n}[\mathrm{~s}]$ & EQUAD per backend-receiver system & Log-uniform $[-8.5,-5]$ & Single-pulsar analysis only \\
\hline$J_{k}[\mathrm{~s}]$ & ECORR per backend-receiver system & Log-uniform $[-8.5,-5]$ & Single-pulsar analysis only \\
\hline \multicolumn{4}{|c|}{ Red noise } \\
\hline$A_{\text {red }}$ & Red-noise power-law amplitude & Log-uniform $[-20,-11]$ & One parameter per pulsar \\
\hline$\gamma_{\text {red }}$ & Red-noise power-law spectral index & Uniform $[0,7]$ & One parameter per pulsar \\
\hline \multicolumn{4}{|c|}{ Phase transition } \\
\hline$T_{*}[\mathrm{GeV}]$ & Phase transition temperature & Log-uniform $[-4,3]$ & One parameter for PTA \\
\hline$\alpha_{*}$ & Phase transition strength & Log-uniform $[-1.3,1]$ & One parameter for PTA \\
\hline$H_{*} / \beta$ & Bubble nucleation rate & Log-uniform $[-2,0]$ & One parameter for PTA \\
\hline$v_{w}$ & Bubble wall velocity & Log-uniform $[-2,1]$ & One parameter for PTA \\
\hline \multicolumn{4}{|c|}{ Supermassive black bole binaries (SMBHBs) } \\
\hline$A_{\mathrm{GWB}}$ & Common process strain amplitude & Log-uniform $[-18,-14]$ & One parameter for PTA \\
\hline$\underline{\gamma_{\mathrm{GWB}}}$ & Common process power-law spectral index & Delta function $\left(\gamma_{\mathrm{GWB}}=13 / 3\right)$ & Fixed \\
\hline
\end{tabular}

lower velocities and the results extrapolated to larger values. This is the approach taken in Refs. [62,63], where the authors show that at high frequencies the GW spectrum is much steeper than predicted by the envelope approximation $(b \sim 1.4-2.3$ depending on the form of the scalar field potential). An alternative approach to the problem has been taken by the authors of Refs. [64,65]. In these works a parametric form for the evolution of the scalar field during bubble collisions is found by using two-bubble simulations. This parametric form is then used in many-bubble simulations to derive the GW spectrum. They also find a steeper high frequency slope $(b \sim 2.6-2.9)$ compared to the prediction of the envelope approximation. Similar discrepancies are found at low frequencies, where both the numerical and semianalytic results find a shallower spectrum compared to the envelope approximation (see Table II). To probe the theoretical uncertainty associated with the bubble contribution, we will carry out three separate BO analyses utilizing each approach and compare the constraint on the phase transition temperature and strength.

Results.-We now report our results for the BO and SWO analyses. For either of them we consider both the case where the only GW signal is the one produced by the $\mathrm{PT}$, and the one in which the PT signal is superimposed to an astrophysical background produced by SMBHB. This latter analyses will give an indication of how difficult it will be to disentangle a signal from a phase transition from the
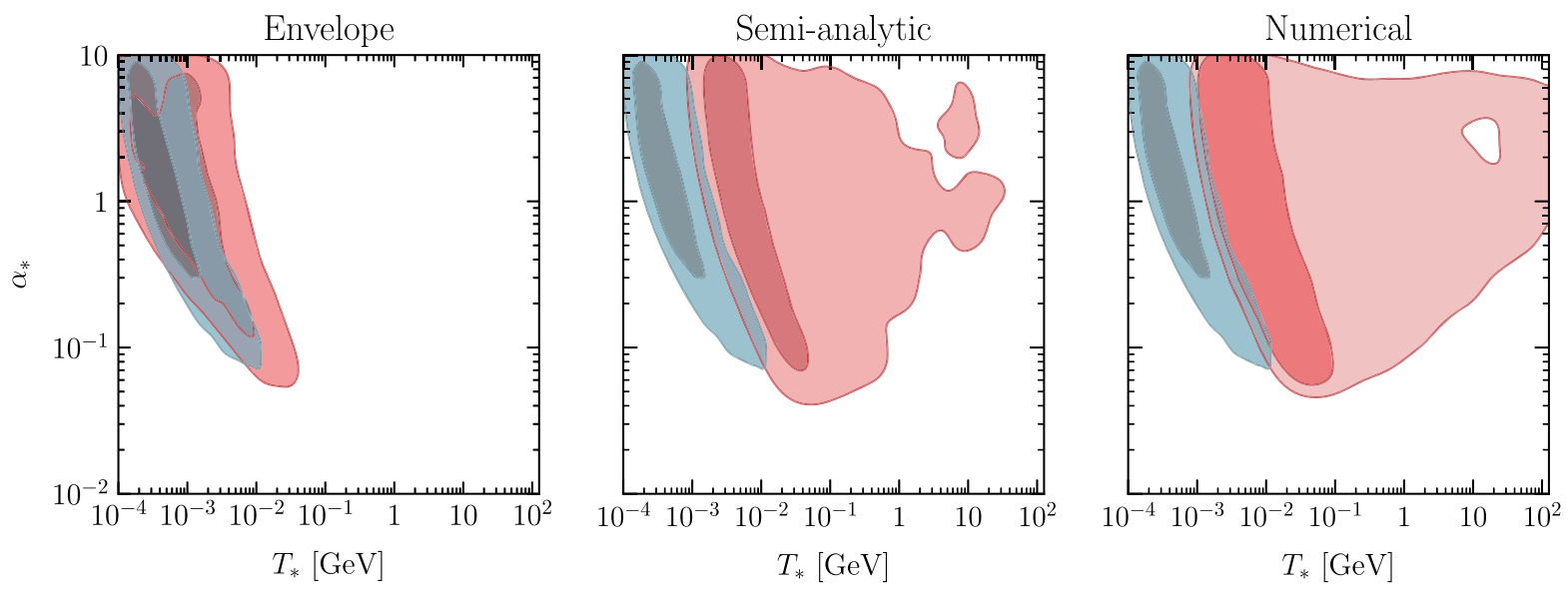

FIG. 1. In red (blue) the $1-\sigma(68 \%$ posterior credible level), and $2-\sigma(95 \%$ posterior credible level) contours for the two-dimensional posterior distributions in the $\left(T_{*}, \alpha_{*}\right)$ plane obtained in the BO (SWO). The BO analysis has been performed with the spectral shape computed by using the envelope approximation (left panel), semianalytic results (central panel), and numerical results (right panel). Specifically, we use $(a, b, c)=(1,2.61,1.5)$ for the semianalytic results, and $(a, b, c)=(0.7,2.3,1)$ for the numerical results. 
SMBHB background. The prior distributions for the model parameters of all these analyses, in addition to other noise characterization parameters, are listed in Table III.

The two parameters that we can constrain the most are the transition temperature, $T_{*}$, and the phase transition strength, $\alpha_{*}$. Their 2D posterior distributions for the PTonly searches are shown in Fig. 1. To assess the impact of theoretical uncertainties related to the bubble spectrum, for the $\mathrm{BO}$ analysis we report the results obtained by using the three different estimates of the bubble contribution to the GW spectrum described in the previous section (envelope, semianalytic, and numerical). We can see that at the 1- $\sigma$ (68\% posterior credible) level all the searches prefer a strong PT, $\alpha_{*} \gtrsim 0.1$, with low transition temperature, $T_{*} \lesssim 10 \mathrm{MeV}$. At $2-\sigma$ (95\% posterior credible) level the posteriors for the semianalytical and numerical results have support at much higher temperatures, while the envelope results still prefer relatively low values. The preference for small values of $T_{*}$ at the 1- $\sigma$ level can be understood by noticing (see Fig. 2) that the data prefer GW spectra that are peaked at frequencies below the NANOGrav sensitivity window (i.e., $f_{*}^{0} \lesssim 10^{-9} \mathrm{~Hz}$ ). And, by setting $\beta / H_{*}=1$ in Eq. (5), we see that this requirement corresponds to $T_{*} \lesssim 10 \mathrm{MeV}$. The low-frequency part of the numerical

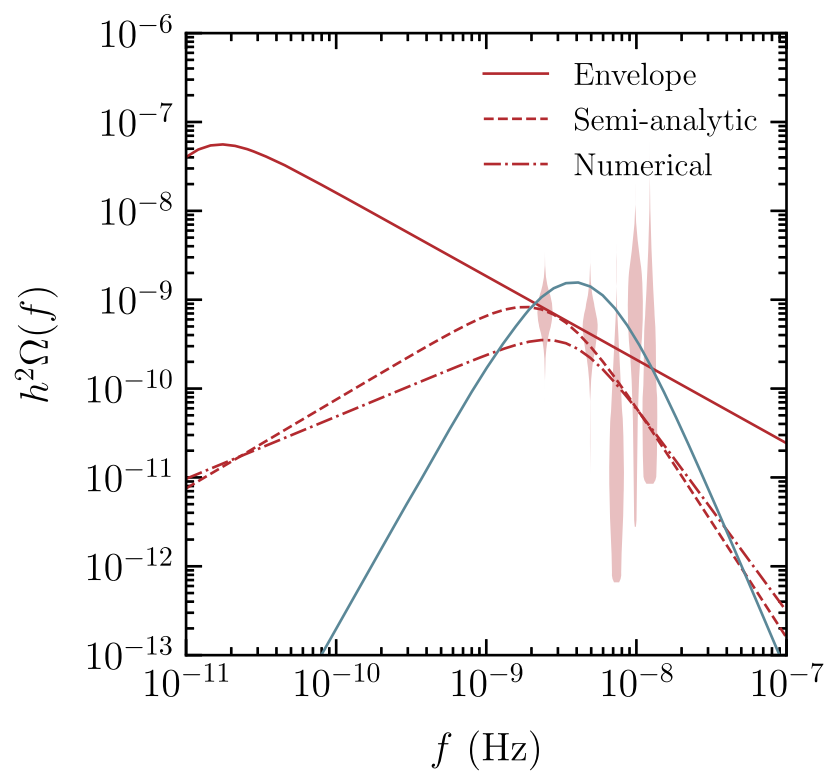

FIG. 2. Maximum likelihood GWB fractional energy-density spectrum for the BO (red) and SWO (blue) analyses compared with the marginalized posterior for the free power spectrum (independent per-frequency characterization; red violin plot) derived in NG12gwb For the BO analysis we show the results derived by using the envelope (solid line), semianalytic (dashed), and numerical (dot-dashed) spectral shapes. For the BO analyses the values of $\left(\alpha_{*}, T_{*}\right)$ for these maximum likelihood spectra are $(0.28,0.7 \mathrm{MeV})$ for the envelope results, $(1.2,3.4 \mathrm{MeV})$ for the semianalytic results, and $(0.13,14.1 \mathrm{MeV})$ for the numerical results. While for the $\mathrm{SO}$ analysis we get $(6.0,0.32 \mathrm{MeV})$. and semianalytical GW spectra is shallow enough that, at the 2- $\sigma$ level, the data can be fitted also by spectra with peak frequencies above the NANOGrav sensitivity window. The same is not true for the envelope results, which have a much steeper low-frequency spectrum; this is the reason why the 2- $\sigma$ levels of the envelope results deviate substantially from the other two.

In Fig. 2 we show the GWB spectrum predicted for the maximum likelihood parameters of PT-only searches. To better illustrate our results, and how the different parameters and theoretical uncertainties affect the GWB spectrum, we release an interactive version of Fig. 2 at this link [66].

To understand how the inclusion of the SMBHB background affects our results, in Fig. 3 we show the posterior for the parameters $\alpha_{*}$ and $A_{\mathrm{GWB}}$ obtained in the PT + SMBHB search. As expected, with the inclusion of the SMBHB background, the posteriors for $\alpha_{*}$ stretch to lower values where most of the signal is provided by the SMBHB contribution [67]. The Bayesian information criterion (BIC) [68], defined to be BIC $=k \ln n-2 \ln \hat{\mathcal{L}}$ where $n=$ 5 is the number of data points in the frequency space, $k$ is the number of parameters in the model and $\hat{\mathcal{L}}$ is the maximum likelihood, is also computed. For the BO searches, the differences in the BIC between the PT + SMBHB and SMBHB only searches are found to be -0.92 , 3.04, and 1.89 for the envelope, semianalytic, and numerical results, respectively; similarly the BIC differences

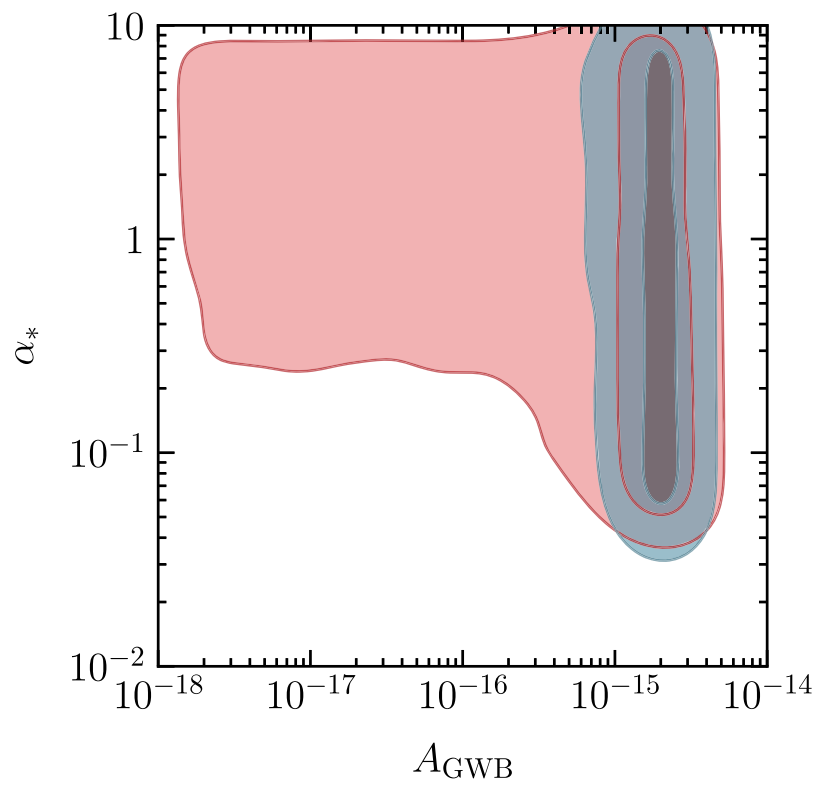

FIG. 3. $1-\sigma(68 \%$ posterior credible level), and $2-\sigma(95 \%$ posterior credible level) contours for the parameters $A_{\mathrm{GWB}}$ and $\alpha_{*}$ in the PT + SMBHB search. In red (blue) the results for the $\mathrm{BO}$ (SWO) analyses. In this figure we have used the semianalytic results for the bubble spectrum. The posteriors do not extend to lower values of $\alpha_{*}$ because of our choice for the $\alpha_{*}$ prior: $\log$ uniform $[-1.3,1]$. 
between the PT-only and SMBHB-only searches are -1.82 , $-3.18,-1.28$. For the SWO analysis, the difference in the BIC between the PT + SMBHB and SMBHB only searches is -4.56 , while we get -2.19 for the difference between the PT-only and SMBHB-only searches. We can then conclude that the PT + SMBHB and PT-only models were neither strongly favored nor disfavored compared to the SMBHB only model [69].

A complete set of posteriors for the parameters of the PTonly searches (derived by using the semianalytic spectrum for the BO analysis) are shown in Fig. 4. As noted previously, at 1- $\sigma$ level the data prefer a strongly firstorder phase transition $\left(\alpha_{*} \gtrsim 0.1\right)$ taking place at temperature $T_{*} \lesssim 10 \mathrm{MeV}$; while no strong constraints on $v_{w}$ or $H_{*} / \beta$ are observed. We can also notice that the higher values of $T_{*}$ allowed in the 2- $\sigma$ region are accompanied by slower nucleation rates (large $H_{*} / \beta$ ). We should caution, however, that numerical simulations have been performed for phase transition strengths up to $\alpha_{*} \sim 0.5$ [70], and that our results for $\alpha_{*} \gtrsim 0.5$ are derived by extrapolating the
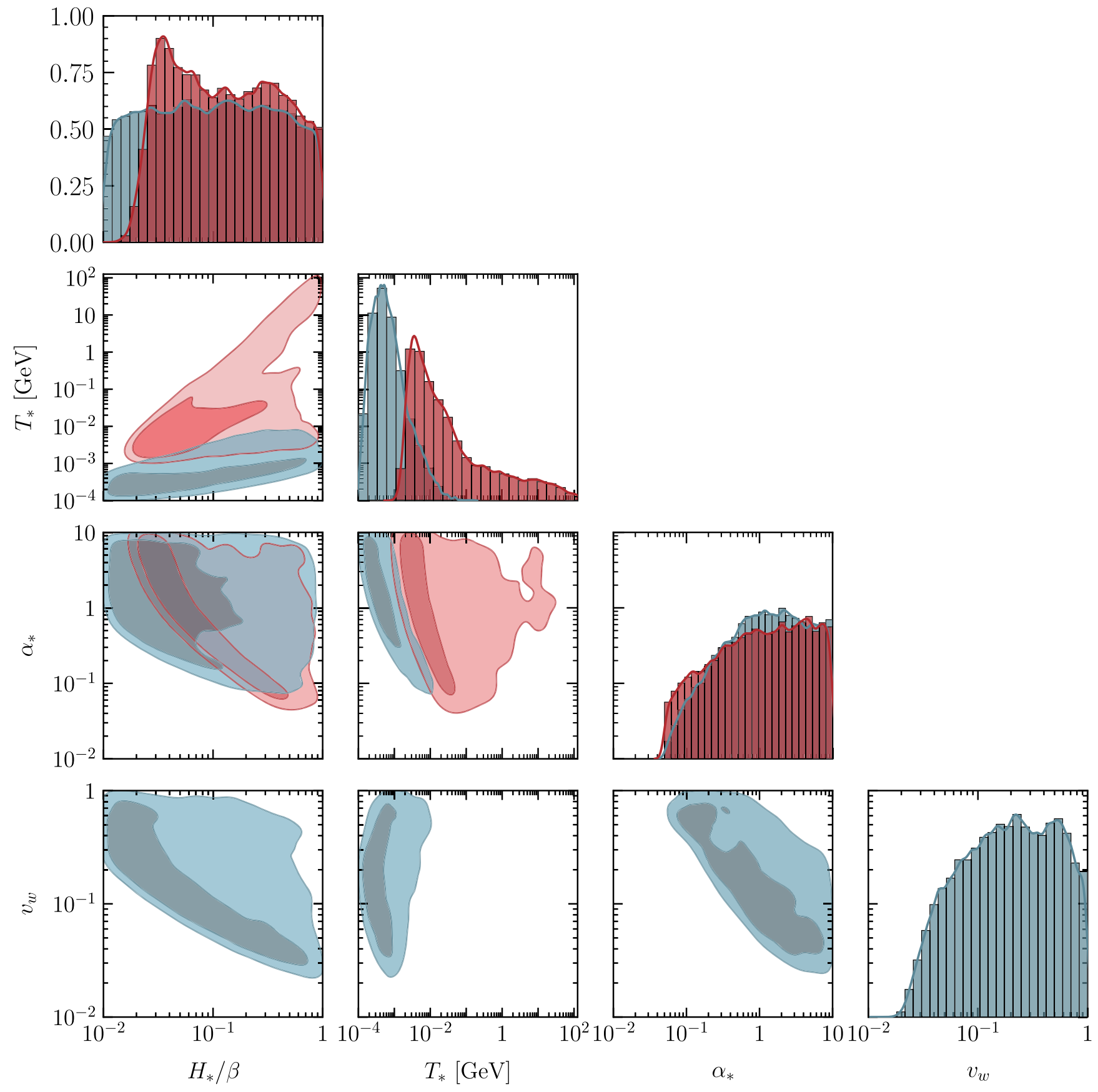

FIG. 4. Corner plot showing the 1D and 2D posterior distributions for the parameters of the PT-only search. In red (blue) the results for the BO (SWO) analyses. In deriving these results we have used the semianalytic bubble spectral shape with $(a, b, c)=(1,2.61,1.5)$. 
results of these simulations. A similar remark should be made for $H_{*} / \beta$, numerical simulations with values of this parameter close to unity have not been performed yet.

Given the low value of $T_{*}$, and the strong constraints on new physics at such low scales, we expect the phase transition to take place in a dark sector with only feeble interactions with the standard model (SM). In order to be consistent with the Hubble parameter constraints during the era of big bang nucleosynthesis (BBN) [71], the energy of this dark sector must be transferred to the SM before the onset of BBN at $T \sim 1 \mathrm{MeV}$. This leaves an allowed range of values for the transition temperature given by $T_{*} \sim 1 \mathrm{MeV}-100 \mathrm{GeV}$. The next data release, which adds multiple years of observations and extends the sensitivity window to lower frequency, should begin to resolve the peak of the spectrum or additionally shrink the range of allowed values for $T_{*}$.

Conclusions. - We performed a search for a stochastic gravitational wave background from first-order phase transitions in the 12.5-year NANOGrav dataset. While previous NANOGrav analysis found no evidence yet for the interpulsar correlation signature of a GWB, the evidence for a common-spectrum process was significant. We found that the data can be modeled by a strong $\left(\alpha_{*}>0.1\right)$ phase transition taking place at temperatures below the electroweak scale. However, the data do not show any strong preference between a SMBHB and a PT generated signal, but we expect to gain additional discriminating power with future datasets, improving the signal to noise ratio and extending the sensitivity window to lower frequencies. In particular, data from the International Pulsar Timing Array will allow the baseline of observations to be significantly extended, and the number of monitored pulsars to be greatly expanded. The present quality of the data is such that our results are not strongly affected by theoretical uncertainties on the GW spectral shape. However, methodological improvements on determining the origin of the GWB spectrum will be needed for future datasets in order to separate the signal from a first-order PT from the SMBHB background, as well as to constrain the microscopic origins of the PT.

Facilities-Arecibo, GBT Software-ENTERPRISE [72], enterprise_extensions [73], HASASIA [74], libstempo [75], matplotlib [76], PTMCMC [77], tempo [78], tempo2 [79], PINT [80].

This work has been carried out by the NANOGrav Collaboration, which is part of the International Pulsar Timing Array. The NANOGrav project receives support from National Science Foundation (NSF) Physics Frontiers Center Grant No. 1430284. The Arecibo Observatory is a facility of the NSF operated under cooperative agreement (No. AST-1744119) by the University of Central Florida (UCF) in alliance with Universidad Ana G. Méndez (UAGM) and Yang Enterprises (YEI), Inc. The Green Bank Observatory is a facility of the NSF operated under cooperative agreement by Associated Universities, Inc. The National Radio Astronomy Observatory is a facility of the NSF operated under cooperative agreement by Associated Universities, Inc. A majority of the computational work was performed on the Caltech High Performance Cluster, partially supported by a grant from the Gordon and Betty Moore Foundation. This work made use of the Super Computing System (Spruce Knob) at WVU, which is funded in part by the National Science Foundation EPSCoR Research Infrastructure Improvement Cooperative Agreement No. 1003907, the state of West Virginia (WVEPSCoR via the Higher Education Policy Commission), and WVU. Part of this research was carried out at the Jet Propulsion Laboratory, California Institute of Technology, under a contract with the National Aeronautics and Space Administration. Portions of this work performed at N. R. L. were supported by Office of Naval Research 6.1 funding. The Flatiron Institute is supported by the Simons Foundation. Pulsar research at U. B. C. is supported by an NSERC Discovery Grant and by the Canadian Institute for Advanced Research. S. R. T. acknowledges support from NSF Grant No. AST-\#2007993, and a Dean's Faculty Fellowship from Vanderbilt University's College of Arts \& Science. J. S. and M. V. acknowledge support from the JPL RTD program. S. B. S. acknowledges support for this work from NSF Grants No. 1458952 and No. 1815664. S. B. S. is a CIFAR Azrieli Global Scholar in the Gravity and the Extreme Universe program. T. T. P. acknowledges support from the MTA-ELTE Extragalactic Astrophysics Research Group, funded by the Hungarian Academy of Sciences (Magyar Tudományos Akadémia), that was used during the development of this research. T. D. and M. L. acknowledge NSF AAG Grant No. 2009468. This work is supported in part by NASA under Grant No. 80GSFC17M0002. V.L., A. M., and K.Z. are supported by the U.S. Department of Energy, Office of Science, Office of High Energy Physics, under Award No. DE-SC0021431 and a Simons Investigator award.

An alphabetical-order author list was used for this Letter in recognition of the fact that a large, decade timescale project such as NANOGrav is necessarily the result of the work of many people. All authors contributed to the activities of the NANOGrav Collaboration leading to the work presented here, and reviewed the manuscript, text, and figures prior to the Letter's submission. Additional specific contributions to this Letter are as follows. Z. A., H. B., P. R. B., H. T. C., M. E. D., P. B. D., T. D., J. A. E., R. D. F., E. C. F., E. F., N. G.-D., P. A. G., D. C. G., M. L. J., M. T. L., D. R. L., R. S. L., J. L., M. A. M., C. N., D. J. N., T. T. P., N. S. P., S. M. R., K. S., I. H. S., R. S., J. K. S., R. S., and S. J. V. developed the 12.5-year dataset through a combination of observations, arrival time calculations, data checks and refinements, and timing model development and analysis; additional specific contributions to the dataset are summarized in NG12. K.Z. and S.R.T. 
coordinated the writing of the Letter. V.L. and A.M. performed all analyses presented in this Letter. K.Z., S. R. T., A. M., and V.L. wrote the Letter and collected the bibliography.

Note added in the proof.-During the revision of this manuscript, the PPTA collaboration published their results for a search of GWs from first-order phase transitions [81]. In that work, they took a different approach compared to our analysis and considered the common signal to be a noise component of unknown origin and derived constraints on the phase transition parameters.

*Also at U.S. Naval Research Laboratory, Washington, D.C. 20375, USA.

[1] R. S. Foster and D. C. Backer, Constructing a pulsar timing array, Astrophys. J. 361, 300 (1990).

[2] S. Detweiler, Pulsar timing measurements and the search for gravitational waves, Astrophys. J. 234, 1100 (1979).

[3] M. V. Sazhin, Opportunities for detecting ultralong gravitational waves, Sov. Astron. 22, 36 (1978).

[4] S. Ransom, A. Brazier, S. Chatterjee, T. Cohen, J. M. Cordes, M. E. DeCesar, P. B. Demorest, J. S. Hazboun, M. T. Lam, R. S. Lynch, M. A. McLaughlin, S. M. Ransom, X. Siemens, S. R. Taylor, and S. J. Vigeland, The NANOGrav program for gravitational waves and fundamental physics, Bull. Am. Astron. Soc. 51, 195 (2019).

[5] G. Desvignes et al., High-precision timing of 42 millisecond pulsars with the European Pulsar Timing Array, Mon. Not. R. Astron. Soc. 458, 3341 (2016).

[6] M. Kerr et al., The Parkes Pulsar Timing Array Project: Second data release, Pub. Astron. Soc. Aust. 37, e020 (2020).

[7] B. C. Joshi, P. Arumugasamy, M. Bagchi, D. Bandyopadhyay, A. Basu, N. Dhanda Batra, S. Bethapudi, A. Choudhary, K. De, L. Dey, A. Gopakumar, Y. Gupta, M. A. Krishnakumar, Y. Maan, P. K. Manoharan, A. Naidu, R. Nandi, D. Pathak, M. Surnis, and A. Susobhanan, Precision pulsar timing with the ORT and the GMRT and its applications in pulsar astrophysics, J. Astrophys. Astron. 39, 51 (2018).

[8] B. B. P. Perera et al., The International Pulsar Timing Array: Second data release, Mon. Not. R. Astron. Soc. 490, 4666 (2019).

[9] K. J. Lee, Prospects of gravitational wave detection using Pulsar Timing Array for Chinese Future Telescopes, in Proceeding sof the Frontiers in Radio Astronomy and FAST Early Sciences Symposium 2015, Astronomical Society of the Pacific Conference Series Vol. 502, edited by L. Qain and D. Li (Astronomical Society of the Pacific, 2016), p. 19, https://myasp.astrosociety.org/product/CS502/vol-502frontiers-in-radio-astronomy-and-fast-early-sciencessymposium-2015.

[10] M. Bailes et al., MeerTime: The MeerKAT key science program on pulsar timing, Proc. Sci., MeerKAT2016 (2018) 011 [arXiv:1803.07424].

[11] C. Ng, Pulsar science with the CHIME telescope, in Pulsar Astrophysics the Next Fifty Years, IAU Symposium Vol. 337, edited by P. Weltevrede, B. B. P. Perera, L. L. Preston, and S.
Sanidas (Cambridge University Press, 2018) pp. 179-182, 10.1017/S1743921317010638.

[12] A. Sesana, F. Haardt, P. Madau, and M. Volonteri, Lowfrequency gravitational radiation from coalescing massive black hole binaries in hierarchical cosmologies, Astrophys. J. 611, 623 (2004).

[13] S. Burke-Spolaor, S. R. Taylor, M. Charisi, T. Dolch, J. S. Hazboun, A. M. Holgado, L. Z. Kelley, T. J. W. Lazio, D. R. Madison, N. McMann, C. M. F. Mingarelli, A. Rasskazov, X. Siemens, J. J. Simon, and T. L. Smith, The astrophysics of nanohertz gravitational waves, Astron. Astrophys. Rev. 27, 5 (2019).

[14] X. Siemens, V. Mandic, and J. Creighton, GravitationalWave Stochastic Background from Cosmic Strings, Phys. Rev. Lett. 98, 111101 (2007).

[15] J. J. Blanco-Pillado, K. D. Olum, and X. Siemens, New limits on cosmic strings from gravitational wave observation, Phys. Lett. B 778, 392 (2018).

[16] L. P. Grishchuk, Amplification of gravitational waves in an isotropic universe, Sov. J. Exp. Theor. Phys. 40, 409 (1975).

[17] P. D. Lasky et al., Gravitational-Wave Cosmology across 29 Decades in Frequency, Phys. Rev. X 6, 011035 (2016).

[18] S. Vagnozzi, Implications of the NANOGrav results for inflation, Mon. Not. R. Astron. Soc. 502, L11 (2021).

[19] J. Winicour, Gravitational radiation from relativistic phase transitions, Astrophys. J. 182, 919 (1973).

[20] C. J. Hogan, Gravitational radiation from cosmological phase transitions, Mon. Not. R. Astron. Soc. 218, 629 (1986).

[21] D. V. Deryagin, D. Y. Grigoriev, V. A. Rubakov, and M. V. Sazhin, Possible anisotropic phases in the early Universe and gravitational wave background, Mod. Phys. Lett. A 01, 593 (1986).

[22] C. Caprini, R. Durrer, and X. Siemens, Detection of gravitational waves from the QCD phase transition with pulsar timing arrays, Phys. Rev. D 82, 063511 (2010).

[23] A. Kobakhidze, C. Lagger, A. Manning, and J. Yue, Gravitational waves from a supercooled electroweak phase transition and their detection with pulsar timing arrays, Eur. Phys. J. C 77, 570 (2017).

[24] M.F. Alam et al., The NANOGrav 12.5-year data set: Observations and narrowband timing of 47 millisecond pulsars, Astrophys. J. Suppl. Ser. 252, 4 (2021).

[25] Z. Arzoumanian et al., and The Nanograv Collaboration, The NANOGrav $12.5 \mathrm{yr}$ data set: Search for an isotropic stochastic gravitational-wave background, Astrophys. J. Lett. 905, L34 (2020).

[26] H. Middleton, A. Sesana, S. Chen, A. Vecchio, W. Del Pozzo, and P. A. Rosado, Massive black hole binary systems and the NANOGrav $12.5 \mathrm{yr}$ results, Mon. Not. R. Astron. Soc. 502, L99 (2021).

[27] N.S. Pol et al., Astrophysics milestones for pulsar timing array gravitational wave detection, Astrophys. J. Lett. 911, L34 (2021).

[28] V. Vaskonen and H. Veermäe, Did NANOGrav See a Signal from Primordial Black Hole Formation?, Phys. Rev. Lett. 126, 051303 (2021).

[29] V. De Luca, G. Franciolini, and A. Riotto, Nanograv Data Hints at Primordial Black Holes as Dark Matter, Phys. Rev. Lett. 126, 041303 (2021). 
[30] J. Ellis and M. Lewicki, Cosmic String Interpretation of Nanograv Pulsar Timing Data, Phys. Rev. Lett. 126, 041304 (2021).

[31] S. Blasi, V. Brdar, and K. Schmitz, Has NANOGrav Found First Evidence for Cosmic Strings?, Phys. Rev. Lett. 126, 041305 (2021).

[32] A. Brandenburg, E. Clarke, Y. He, and T. Kahniashvili, Can we observe the QCD phase transition-generated gravitational waves through pulsar timing arrays?, arXiv: 2102.12428.

[33] A. Neronov, A. R. Pol, C. Caprini, and D. Semikoz, NANOGrav signal from magnetohydrodynamic turbulence at the QCD phase transition in the early Universe, Phys. Rev. D 103, L041302 (2021).

[34] S.-L. Li, L. Shao, P. Wu, and H. Yu, NANOGrav signal from first-order confinement/deconfinement phase transition in different QCD matters, Phys. Rev. D 104, 043510 (2021).

[35] B. Barman, A. Dutta Banik, and A. Paul, Implications of NANOGrav results and UV freeze-in in a fast-expanding Universe, arXiv:2012.11969.

[36] K. T. Abe, Y. Tada, and I. Ueda, Induced gravitational waves as a cosmological probe of the sound speed during the QCD phase transition, J. Cosmol. Astropart. Phys. 06 (2021) 048.

[37] W. Ratzinger and P. Schwaller, Whispers from the dark side: Confronting light new physics with NANOGrav data, SciPost Phys. 10, 047 (2021).

[38] A. Addazi, Y.-F. Cai, Q. Gan, A. Marciano, and K. Zeng, NANOGrav results and dark first order phase transitions, Sci. China Phys. Mech. Astron. 64, 290411 (2021).

[39] Y. Nakai, M. Suzuki, F. Takahashi, and M. Yamada, Gravitational waves and dark radiation from dark phase transition: Connecting NANOGrav pulsar timing data and Hubble tension, Phys. Lett. B 816, 136238 (2021).

[40] M. J. Strassler and K. M. Zurek, Echoes of a hidden valley at hadron colliders, Phys. Lett. B 651, 374 (2007).

[41] Z. Chacko, L. J. Hall, and Y. Nomura, Acceleressence: Dark energy from a phase transition at the seesaw scale, J. Cosmol. Astropart. Phys. 10 (2004) 011.

[42] P. Schwaller, Gravitational Waves from a Dark Phase Transition, Phys. Rev. Lett. 115, 181101 (2015).

[43] M. Battaglieri et al., US cosmic visions: New ideas in dark matter 2017: Community report, arXiv:1707.04591.

[44] S.-L. Li, L. Shao, P. Wu, and H. Yu, NANOGrav signal from first-order confinement/deconfinement phase transition in different QCD matters, Phys. Rev. D 104, 043510 (2021).

[45] L. Bian, R.-G. Cai, J. Liu, X.-Y. Yang, and R. Zhou, On the gravitational wave sources from the NANOGrav 12.5-yr data, Phys. Rev. D 103, L081301 (2021).

[46] C. J. Moore, R. H. Cole, and C. P. L. Berry, Gravitationalwave sensitivity curves, Classical Quantum Gravity 32, 015014 (2015).

[47] N. Aghanim et al. (Planck Collaboration), Planck 2018 results. VI. Cosmological parameters, Astron. Astrophys. 641, A6 (2020).

[48] E. S. Phinney, A practical theorem on gravitational wave backgrounds, arXiv:astro-ph/0108028.

[49] R. W. Hellings and G. S. Downs, Upper limits on the isotropic gravitational radiation background from pulsar timing analysis, Astrophys. J. Lett. 265, L39 (1983).
[50] C. Caprini et al., Science with the space-based interferometer eLISA. II: Gravitational waves from cosmological phase transitions, J. Cosmol. Astropart. Phys. 04 (2016) 001.

[51] A. Roper Pol, S. Mandal, A. Brandenburg, T. Kahniashvili, and A. Kosowsky, Numerical simulations of gravitational waves from early-universe turbulence, Phys. Rev. D 102, 083512 (2020).

[52] A. Brandenburg, E. Clarke, Y. He, and T. Kahniashvili, Can we observe the QCD phase transition-generated gravitational waves through pulsar timing arrays?, Phys. Rev. D 104, 043513 (2021).

[53] P. Binetruy, A. Bohe, C. Caprini, and J.-F. Dufaux, Cosmological backgrounds of gravitational waves and eLISA/NGO: Phase transitions, cosmic strings and other sources, J. Cosmol. Astropart. Phys. 06 (2012) 027.

[54] H.-K. Guo, K. Sinha, D. Vagie, and G. White, Phase transitions in an expanding universe: Stochastic gravitational waves in standard and non-standard histories, J. Cosmol. Astropart. Phys. 01 (2021) 001.

[55] J. Ellis, M. Lewicki, and J. M. No, Gravitational waves from first-order cosmological phase transitions: Lifetime of the sound wave source, J. Cosmol. Astropart. Phys. 07 (2020) 050.

[56] D. J. Weir, Gravitational waves from a first order electroweak phase transition: A brief review, Phil. Trans. R. Soc. A 376, 20170126 (2018).

[57] M. Hindmarsh and M. Hijazi, Gravitational waves from first order cosmological phase transitions in the Sound Shell Model, J. Cosmol. Astropart. Phys. 12 (2019) 062.

[58] R. Jinno and M. Takimoto, Gravitational waves from bubble collisions: An analytic derivation, Phys. Rev. D 95, 024009 (2017).

[59] M. Hindmarsh, S. J. Huber, K. Rummukainen, and D. J. Weir, Shape of the acoustic gravitational wave power spectrum from a first order phase transition, Phys. Rev. D 96, 103520 (2017); 101, 089902(E) (2020).

[60] J. R. Espinosa, T. Konstandin, J. M. No, and G. Servant, Energy budget of cosmological first-order phase transitions, J. Cosmol. Astropart. Phys. 06 (2010) 028.

[61] A. Kosowsky, M. S. Turner, and R. Watkins, Gravitational radiation from colliding vacuum bubbles, Phys. Rev. D 45, 4514 (1992).

[62] D. Cutting, E. G. Escartin, M. Hindmarsh, and D. J. Weir, Gravitational waves from vacuum first order phase transitions II: From thin to thick walls, Phys. Rev. D 103, 023531 (2021).

[63] D. Cutting, M. Hindmarsh, and D. J. Weir, Gravitational waves from vacuum first-order phase transitions: From the envelope to the lattice, Phys. Rev. D 97, 123513 (2018).

[64] M. Lewicki and V. Vaskonen, Gravitational waves from colliding vacuum bubbles in gauge theories, Eur. Phys. J. C 81, 437 (2021).

[65] M. Lewicki and V. Vaskonen, Gravitational wave spectra from strongly supercooled phase transitions, Eur. Phys. J. C 80, 1003 (2020).

[66] https://mybinder.org/v2/gh/AMitridate/bokeh/master? urlpath=\%2Fproxy $\% 2$ F5006\%2Fbokeh-app.

[67] The posterior stops around $\alpha_{*} \simeq 0.05$ because of choice of the prior, otherwise it would extend down to $\alpha_{*} \sim 0$.

[68] G. Schwarz, Estimating the dimension of a model, Ann. Stat. 6, 461 (1978). 
[69] R. E. Kass and A. E. Raftery, Bayes factors, J. Am. Stat. Assoc. 90, 773 (1995).

[70] D. Cutting, M. Hindmarsh, and D. J. Weir, Vorticity, Kinetic Energy, and Suppressed Gravitational Wave Production in Strong First-Order Phase Transitions, Phys. Rev. Lett. 125, 021302 (2020).

[71] P. A. Zyla et al. (Particle Data Group), Review of particle physics, Prog. Theor. Exp. Phys. 2020, 083 C01 (2020).

[72] J. A. Ellis, M. Vallisneri, S. R. Taylor, and P.T. Baker, ENTERPRISE: Enhanced numerical toolbox enabling a robust PulsaR inference SuitE (2019), ascl:1912.015, https://ui .adsabs.harvard.edu/abs/2019ascl.soft12015E/abstract.

[73] S. R. Taylor, P. T. Baker, J. S. Hazboun, J. J. Simon, and S. J. Vigeland, enterprise_extensions (2021), https://github.com/ nanograv/enterprise_extensions

[74] J. Hazboun, J. Romano, and T. Smith, Hasasia: A Python package for pulsar timing array sensitivity curves, J. Open Source Software 4, 1775 (2019).

[75] M. Vallisneri, libstempo: Python wrapper for Tempo2 (2020), ascl:2002.017, https://ui.adsabs.harvard.edu/abs/ 2020ascl.soft02017V/abstract.
[76] J.D. Hunter, Matplotlib: A 2D graphics environment, Comput. Sci. Eng. 9, 90 (2007).

[77] J. Ellis and R. van Haasteren, jellis18/ptmcmcsampler: Official release (2017) 10.5281/zenodo.1037579.

[78] D. Nice, P. Demorest, I. Stairs, R. Manchester, J. Taylor, W. Peters, J. Weisberg, A. Irwin, N. Wex, and Y. Huang, Tempo: Pulsar timing data analysis (2015), ascl:1509.002, https://ui.adsabs.harvard.edu/abs/2015ascl.soft09002N/ abstract.

[79] G. Hobbs and R. Edwards, Tempo2: Pulsar timing package (2012), ascl:1210.015, https://ui.adsabs.harvard.edu/abs/ 2012ascl.soft10015H/abstract.

[80] J. Luo, S. Ransom, P. Demorest, R. van Haasteren, P. Ray, K. Stovall, M. Bachetti, A. Archibald, M. Kerr, J. Colen, and F. Jenet, PINT: High-precision pulsar timing analysis package (2019), ascl:1902.007, https://ui.adsabs.harvard .edu/abs/2019ascl.soft02007L/abstract.

[81] X. Xue et al., following Letter, Constraining Cosmological Phase Transitions with the Parkes Pulsar Timing Array, Phys. Rev. Lett. 127, 251303 (2021). 